\title{
Transversals of longest paths*
}

\author{
Márcia R. Cerioli ${ }^{1}$, Cristina G. Fernandes $^{\dagger 2}$, Renzo Gómez ${ }^{\ddagger 2}$, \\ Juan Gutiérrez ${ }^{\$ 2}$, and Paloma T. Lima ${ }^{3}$ \\ ${ }^{1}$ COPPE Sistemas e Instituto de Matemática, Universidade Federal do Rio de \\ Janeiro, Brazil \\ ${ }^{2}$ Departamento de Ciência da Computação, Universidade de São Paulo, Brazil \\ ${ }^{3}$ Department of Informatics, University of Bergen, Norway
}

\begin{abstract}
Let $\operatorname{lpt}(G)$ be the minimum cardinality of a set of vertices that intersects all longest paths in a graph $G$. Let $\omega(G)$ be the size of a maximum clique in $G$, and $\operatorname{tw}(G)$ be the treewidth of $G$. We prove that $\operatorname{lpt}(G) \leq \max \{1, \omega(G)-2\}$ when $G$ is a connected chordal graph; that $\operatorname{lpt}(G)=1$ when $G$ is a connected bipartite permutation graph or a connected full substar graph; and that $\operatorname{lpt}(G) \leq \operatorname{tw}(G)$ for any connected graph $G$.
\end{abstract}

\section{INTRODUCTION}

It is a well-known fact that, in a connected graph, any two longest paths have a common vertex. In 1966, Gallai raised the following question: Does every connected graph contain a vertex that belongs to all of its longest paths? The answer to Gallai's question is already known to be negative. Figure 1 shows the smallest known negative example, on 12 vertices, which was independently found by Walther and Voss [19] and Zamfirescu [20]. However, when we restrict ourselves to some specific classes of graphs, the answer to Gallai's question turns out to be positive. For example, it is well known that any set of subtrees of a tree satisfies the Helly property. If we consider the set of subtrees consisting of the longest paths of the tree, since they are pairwise intersecting, we conclude that there is a vertex that belongs to all of them.

There are other graph classes which are known to have a positive answer to Gallai's question. Klavžar and Petkovšek [16] proved that this is the case for split graphs, cacti, and graphs whose blocks are Hamilton-connected, almost Hamilton-connected or cycles. Balister et al. [2] and Joos [15] proved the same for the class of circular arc graphs. De Rezende et al. [7] proved that the answer to Gallai's question is positive for 2-trees and Chen et al. [6] extended this result for series-parallel graphs, also known as partial 2-trees. Chen [5] proved the same for graphs with matching number smaller than three, while Cerioli and Lima [4, 17] proved it for $P_{4}$-sparse graphs, $\left(P_{5}, K_{1,3}\right)$-free graphs, graphs that are the join of two other graphs and starlike graphs, a superclass of split graphs. Finally, Jobson et al. [14] proved it for dually chordal graphs and Golan and Shan [11] for $2 K_{2}$-free graphs.

\footnotetext{
*A preliminary version of this paper was presented at the IX Latin and American Algorithms, Graphs and Optimization Symposium (LAGOS'17).

${ }^{\dagger}$ Research partially supported by CNPq (Proc. 456792/2014-7 and 308116/2016-0), FAPESP (2013/03447-6 and 2015/08538-5) and Project MaCLinC of NUMEC/USP.

${ }^{\ddagger}$ Research supported by CAPES (Proc. 235671298-48).

\$ Research supported by FAPESP (Proc. 2015/08538-5).
} 


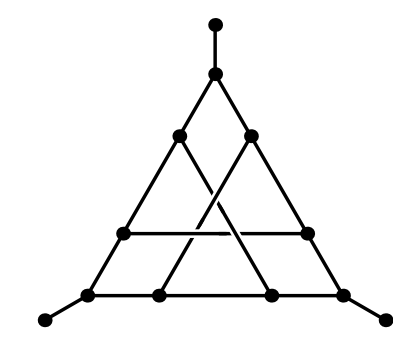

Figure 1: The classical 12-vertex example that has a negative answer to Gallai's question.

A more general approach to Gallai's question is to ask for the size of the smallest transversal of longest paths of a graph, that is, the smallest set of vertices that intersects every longest path. Given a graph $G$, we denote the size of such a set by $\operatorname{lpt}(G)$. In this direction, Rautenbach and Sereni [18] proved that $\operatorname{lpt}(G) \leq\left\lceil\frac{n}{4}-\frac{n^{2 / 3}}{90}\right\rceil$ for every connected graph $G$ on $n$ vertices, that $\operatorname{lpt}(G) \leq 9 \sqrt{n} \log n$ for every connected planar graph $G$ on $n$ vertices, and that $\operatorname{lpt}(G) \leq k+1$ for every connected graph $G$ of treewidth at most $k$.

In this work, we provide exact results and upper bounds on the value of $\operatorname{lpt}(G)$ when $G$ belongs to some specific classes of graphs. More specifically, we prove that:

- $\operatorname{lpt}(G) \leq \max \{1, \omega(G)-2\}$ for every connected chordal graph $G$, where $\omega(G)$ is the size of a maximum clique of $G$.

- $\operatorname{lpt}(G)=1$ for every connected bipartite permutation graph $G$.

- $\operatorname{lpt}(G) \leq k$ for every connected graph $G$ of treewidth at most $k$.

- $\operatorname{lpt}(G)=1$ for every connected full substar graph $G$.

This paper is organized as follows. In the next section, we state the definitions and basic results that are going to be used throughout the text. In Sections [II] IV [V] and VI, we consider, respectively, the class of chordal graphs, the class bipartite permutation graphs, the class of graphs of treewidth at most $k$ and the class of full substar graphs. Finally, in Section VII. we state the open problems to be considered in future work.

\section{DeFINITIONS AND NOTATION}

All graphs considered are simple. Let $u$ be a vertex in a graph $G$, we denote by $N_{G}(u)$ the set of neighbors of $u$ in $G$, and by $d_{G}(u)$ the cardinality of $N_{G}(u)$. If the context is clear, we write simply $d(u)$ and $N(u)$ respectively. Let $P$ be a path in a graph $G$. We denote by $|P|$ the length of $P$, that is, the number of edges in $P$. Given a path $Q$ such that the only vertex it shares with $P$ is an extreme of both of them, we denote by $P$. $Q$ the concatenation of $P$ and $Q$. For a vertex $v$ in $P$, let $P^{\prime}$ and $P^{\prime \prime}$ be the paths such that $P=P^{\prime} \cdot P^{\prime \prime}$ with $P^{\prime} \cap P^{\prime \prime}=\{v\}$. We refer to these two paths as the $v$-tails of $P$. Given a path $P$ that contains vertices $a$ and $b$, we denote by $P_{a}$ the $a$-tail of $P$ that does not contain $b$ and by $P_{b}$ the $b$-tail of $P$ that does not contain $a$. Also, if the context is clear, we denote by $\widetilde{P}$ the subpath of $P$ that has $a$ and $b$ as its extremes. Thus $P=P_{a} \cdot \widetilde{P} \cdot P_{b}$.

Let $S$ be a set of vertices of $G$. Let $P$ be a path in $G$ that does not contain all vertices of $S$ and contains a vertex not in $S$. We say that $S$ fences $P$ if all the vertices of $P-S$ are in a single component of $G-S$, otherwise we say that $P$ crosses $S$. Given a path $P$ that crosses $S$ and has both extremes not in $S$, we say that $P$ is extreme-separated by $S$ when the extremes of $P$ are in different 
components of $G-S$, and that $P$ is extreme-joined by $S$ if its extremes are in the same component of $G-S$.

For an integer $t$, we say that $P$ t-touches $S$ if $P$ intersects $S$ at exactly $t$ vertices. A path $P$ is an $S$-corner path if $P$ 1-touches $S$. Let $P$ be an $S$-corner path. If $P$ is fenced by $S$, we say that $P$ is an $S$-corner-fenced path. If $P$ crosses $S$, we say that $P$ is an $S$-corner-crossing path. If two paths $P$ and $Q$ touch $S$ at the same set of vertices, we say they are $S$-equivalent, otherwise they are S-nonequivalent.

If $P$ is fenced by $S$, we denote by $\operatorname{Comp}_{S}(P)$ the set of vertices of the component of $G-S$ where $P-S$ lies. For a set $X$ of vertices not contained in $S$, we denote by $\operatorname{Comp}_{S}(X)$ the set of vertices of the components of $G-S$ where $X \backslash S$ lies. Two fenced paths $P$ and $Q$ are $S$ component-disjoint if $\operatorname{Comp}_{S}(P) \neq \operatorname{Comp}_{S}(Q)$. If $S$ is clear from the context, we just say they are component-disjoint.

From now on, we use $L=L(G)$ for the length of a longest path in $G$. Also, remember that $\omega(G)$ is the size of a maximum clique of $G$.

A graph $H$ is called a minor of the graph $G$ if $H$ can be formed from $G$ by deleting edges and vertices and by contracting edges.

A tree decomposition [8, p. 337] of a graph $G$ is a pair $(T, \mathcal{V})$, conformed by a tree $T$ and a collection $\mathcal{V}=\left\{V_{t}: t \in V(T)\right\}$ of bags $V_{t} \subseteq V(G)$, that satisfies the following three conditions:

(T1) $\bigcup_{t \in V(T)} V_{t}=V(G)$;

(T2) for every $u v \in E(G)$, there exists a bag $V_{t}$ such that $u, v \in V_{t}$;

(T3) if a vertex $v$ is in two different bags $V_{t_{1}}, V_{t_{2}}$, then $v$ is also in any bag $V_{t}$ such that $t$ is on the (unique) path from $t_{1}$ to $t_{2}$ in $T$.

The width of $(T, \mathcal{V})$ is the number

$$
\max \left\{\left|V_{t}\right|-1: t \in V(T)\right\},
$$

and the treewidth $t w(G)$ of $G$ is the minimum width of any tree decomposition of $G$.

A graph is called chordal if every induced cycle has length three. Next we present some basic properties on tree decompositions for general and chordal graphs. We fix a graph $G$ and a tree decomposition $(T, \mathcal{V})$ of $G$. Proposition 1 is due to Bodlaender [3]. Gross [12] presented a proof for it and refers to tree decompositions such as in Proposition 1 as full tree decompositions. The tree decomposition mentioned in Proposition 2 is also called clique tree and it was introduced by Gavril [10]. Proposition 3 is a direct consequence of Corollary 12.3.12 of the book of Diestel [8].

Proposition 1. If $k$ is the treewidth of a graph $G$, then $G$ has a tree decomposition $(T, \mathcal{V})$ of width $k$ such that $\left|V_{t}\right|=k+1$ for every $t \in V(T)$, and $\left|V_{t} \cap V_{t^{\prime}}\right|=k$ for every $t t^{\prime} \in E(T)$.

Proposition 2. Every chordal graph $G$ has a tree decomposition $(T, \mathcal{V})$ such that the bags of $\mathcal{V}$ are the maximal cliques of $G$.

Proposition 3. For every chordal graph $G, \operatorname{tw}(G)=\omega(G)-1$.

Given two different nodes $t, t^{\prime}$ of $T$, we denote by $\operatorname{Branch}_{t}\left(t^{\prime}\right)$ the component of $T-t$ where $t^{\prime}$ lies. We say that such component is a branch of $T$ at $t$ and that the components of $T-t$ are the branches of $T$ at $t$ [13]. Similarly, for a vertex $v \notin V_{t}$, we denote by $\operatorname{Branch}_{t}(v)$ the branch $\operatorname{Branch}_{t}\left(t^{\prime}\right)$ of $T$ at $t$ such that $v \in V_{t^{\prime}}$. We also say that $v$ is in $\operatorname{Branch}_{t}\left(t^{\prime}\right)$. Moreover, we can extend the notation and say that, if $P$ is a path fenced by $V_{t}$ for some $t \in T$, then $\operatorname{Branch}_{t}(P)=\operatorname{Branch}_{t}(v)$, where $v$ is a vertex of $P-V_{t}$. We also say that $P$ is in $\operatorname{Branch}_{t}(v)$. Next we show some basic propositions of branches. Propositions 4 to 6 are used to justify that the previous two definitions are coherent. The first two of them appear in the work of Heinz [13]. 
Proposition 4. Let $t$ be a node of $T$ and $v$ be a vertex of $G$ such that $v \notin V_{t}$. Let $t^{\prime}$ and $t^{\prime \prime}$ be nodes of $T$. If $v \in V_{t^{\prime}} \cap V_{t^{\prime \prime}}$, then $t^{\prime}$ and $t^{\prime \prime}$ are in the same branch of $T$ at $t$.

Proposition 5. Let $u$ and $v$ be two vertices of $G$, and let $t$ be a node of $T$. If $u, v \notin V_{t}$, and $u$ and $v$ are not separated by $V_{t}$ in $G$, then $\operatorname{Branch}_{t}(u)=\operatorname{Branch}_{t}(v)$.

Proposition 6. Let $t$ be a node of $T$ and $P$ be a path fenced by $V_{t}$. For every two vertices $u$ and $v$ in $P-V_{t}$, $\operatorname{Branch}_{t}(u)=\operatorname{Branch}_{t}(v)$.

Proof. By definition of fenced paths, $u$ and $v$ lie in the same component of $G-V_{t}$, so they are not separated by $V_{t}$ in $G$ and we can apply Proposition 5 .

Proposition 7. If $P$ is a path fenced by $V_{t}$ for some $t \in V(T)$, then there exists a neighbor $t^{\prime}$ of $t$ in $T$ such that $\operatorname{Branch}_{t}(P)=\operatorname{Branch}_{t}\left(t^{\prime}\right)$.

Proof. Let $u$ be a vertex of $P-V_{t}$ (that exists by the definition of fenced). As $u \notin V_{t}$, there exists a bag $V_{t^{\prime \prime}} \neq V_{t}$ that contains $u$. Let $t^{\prime}$ be the neighbor of $t$ in $T$ such that $t^{\prime}$ is in the (unique) path from $t$ to $t^{\prime \prime}$ in $T$. Then $\operatorname{Branch}_{t}(P)=\operatorname{Branch}_{t}(u)=\operatorname{Branch}_{t}\left(t^{\prime \prime}\right)=\operatorname{Branch}_{t}\left(t^{\prime}\right)$.

Proposition 8 appears in the book of Diestel [8] as Lemma 12.3.1. Proposition 9 is a corollary of Proposition 8

Proposition 8. Let $p q \in E(T)$. Let $T_{p}=\operatorname{Branch}_{q}(p)$ and $T_{q}=\operatorname{Branch}_{p}(q)$ be the components of $T-p q$, with $p \in V\left(T_{p}\right)$ and $q \in V\left(T_{q}\right)$. Then $V_{p} \cap V_{q}$ separates $\bigcup_{t \in V\left(T_{p}\right)} V_{t}$ from $\bigcup_{t \in V\left(T_{q}\right)} V_{t}$ in $G$.

Proposition 9. Let $p q \in E(T)$. Let $u$ and $v$ be vertices of $G$ with $v \notin V_{p}$. If $u \in V_{p} \backslash V_{q}$ and $\operatorname{Branch}_{p}(v)=\operatorname{Branch}_{p}(q)$, then $u$ and $v$ are not adjacent.

Proof. Observe that $u$ is in a bag of $\operatorname{Branch}_{q}(p)$ because $p$ is in $\operatorname{Branch}_{q}(p)$ and $u \in V_{p}$. As $v$ is in a bag of $\operatorname{Branch}_{p}(q)$, by Proposition $8, V_{p} \cap V_{q}$ separates $u$ from $v$. Hence, $u$ and $v$ are not adjacent.

Proposition 10. Let $t \in V(T)$. Let $P^{\prime}$ be a path fenced by $V_{t}$ that 1-touches $V_{t}$ such that $\operatorname{Branch}_{t}\left(P^{\prime}\right)=\operatorname{Branch}_{t}\left(t^{\prime}\right)$, where $t t^{\prime} \in E(T)$. Then $V_{t} \cap P^{\prime} \subseteq V_{t^{\prime}}$.

Proof. Suppose by contradiction that there exists a vertex $x \in\left(V_{t} \cap P^{\prime}\right) \backslash V_{t^{\prime}}$. As $P^{\prime}$ is fenced by $V_{t}$, there exists a vertex $y \in P^{\prime}-V_{t}$. Moreover, $\operatorname{Branch}_{t}(y)=\operatorname{Branch}_{t}\left(P^{\prime}\right)=\operatorname{Branch}_{t}\left(t^{\prime}\right)$. Let $P_{x y}^{\prime}$ be the subpath of $P^{\prime}$ with $x$ and $y$ as extremes. Since $P^{\prime}$ 1-touches $V_{t}$, the subpath $P_{x y}^{\prime}$ also 1-touches $V_{t}$. This implies that $P_{x y}^{\prime}$ is internally disjoint from $V_{t}$. As $x, y \notin V_{t} \cap V_{t^{\prime}}$, the subpath $P_{x y}^{\prime}$ is disjoint from $V_{t} \cap V_{t^{\prime}}$. But then we contradict Proposition 8, which says that $V_{t} \cap V_{t^{\prime}}$ separates $x$, which is in a bag of $\operatorname{Branch}_{t^{\prime}}(t)$, from $y$, which is in a bag of $\operatorname{Branch}_{t}\left(t^{\prime}\right)$.

Proposition 11. Let $t \in V(T)$ and $t t^{\prime} \in E(T)$. Let $P^{\prime}$ be a path fenced by $V_{t}$ that 1-touches $V_{t}$ such that $\operatorname{Branch}_{t}\left(P^{\prime}\right)=\operatorname{Branch}_{t}\left(t^{\prime}\right)$. Let $Q^{\prime}$ be a path fenced by $V_{t}$ that 1-touches $V_{t}$ at a vertex in $V_{t} \backslash V_{t^{\prime}}$. Then $P^{\prime} \cap Q^{\prime}=\varnothing$.

Proof. Suppose by contradiction that $P^{\prime} \cap Q^{\prime} \neq \varnothing$. As $P \cap Q \cap V_{t}=\varnothing$, we must have that $\operatorname{Branch}_{t}\left(Q^{\prime}\right)=\operatorname{Branch}_{t}\left(P^{\prime}\right)=\operatorname{Branch}_{t}\left(t^{\prime}\right)$. By Proposition 10, $V_{t} \cap Q^{\prime} \subseteq V_{t^{\prime}}$, a contradiction. 


\section{ChORDAL GRAPHS}

We start by proving a lemma that is valid for every graph.

Lemma 12. Let $G$ be a graph with a clique $K$. Let $\mathscr{C}$ be the set of all longest paths in $G$ that cross $K$, 2 -touch $K$, and are extreme-joined by $K$. There are at most two K-nonequivalent paths in $\mathscr{C}$.

Proof. Suppose by contradiction that there are (at least) three $K$-nonequivalent longest paths $P, Q$, and $R$ in $\mathscr{C}$. Say $P \cap K=\{a, b\}, Q \cap K=\{c, d\}$, and $R \cap K=\{e, f\}$, where $\{a, b\},\{c, d\}$, and $\{e, f\}$ are pairwise distinct but not necessarily pairwise disjoint. We may assume that either $\{a, b\} \cap$ $\{c, d\}=\varnothing$ or $\{a, b\} \cap\{c, d\}=\{b\}=\{d\}$. If $\tilde{P}$ is component-disjoint from $Q_{c}$ (and from $Q_{d}$ ), and $\tilde{Q}$ is component-disjoint from $P_{a}$ (and from $P_{b}$ ), then $P_{a} \cdot a c \cdot \tilde{Q} \cdot d b \cdot P_{b}$ and $Q_{c} \cdot c a \cdot \tilde{P} \cdot b d \cdot Q_{d}$ are paths whose lengths sum more than $2 L$, a contradiction, as at least one of them would have length greater than $L$. So,

$$
\operatorname{Comp}_{K}(\tilde{P})=\operatorname{Comp}_{K}\left(Q_{c}\right) \text { or } \operatorname{Comp}_{K}(\tilde{Q})=\operatorname{Comp}_{K}\left(P_{a}\right)
$$

Applying the same reasoning to paths $P$ and $R$, and to paths $Q$ and $R$, we conclude that

$$
\operatorname{Comp}_{K}(\tilde{P})=\operatorname{Comp}_{K}\left(R_{e}\right) \text { or } \operatorname{Comp}_{K}(\tilde{R})=\operatorname{Comp}_{K}\left(P_{a}\right),
$$

and that

$$
\operatorname{Comp}_{K}(\tilde{Q})=\operatorname{Comp}_{K}\left(R_{e}\right) \text { or } \operatorname{Comp}_{K}(\tilde{R})=\operatorname{Comp}_{K}\left(Q_{c}\right) \text {. }
$$

Also, as $P, Q$, and $R$ cross $K$, from (1), (2), and (3), we have that

$$
\operatorname{Comp}_{K}(\tilde{P}) \neq \operatorname{Comp}_{K}(\tilde{Q}), \operatorname{Comp}_{K}(\tilde{P}) \neq \operatorname{Comp}_{K}(\tilde{R}) \text {, and } \operatorname{Comp}_{K}(\tilde{Q}) \neq \operatorname{Comp}_{K}(\tilde{R}) .
$$

Without loss of generality, we may assume that $\operatorname{Comp}_{K}(\tilde{P})=\operatorname{Comp}_{K}\left(Q_{c}\right)$. (Otherwise, interchange $P$ with $Q$, and $\{a, b\}$ with $\{c, d\}$.) See Figure $2(a)$. Now, if $\operatorname{Comp}_{K}(\tilde{P})=\operatorname{Comp}_{K}\left(R_{e}\right)$, then, by (4), $\operatorname{Comp}_{K}(\tilde{Q}) \neq \operatorname{Comp}_{K}\left(R_{e}\right)$, and thus, by (3), $\operatorname{Comp}_{K}(\tilde{R})=\operatorname{Comp}_{K}\left(Q_{c}\right)$. But then $\operatorname{Comp}_{K}(\tilde{R})=\operatorname{Comp}_{K}(\tilde{P})$, and we contradict (4). Hence, $\operatorname{Comp}_{K}(\tilde{P}) \neq \operatorname{Comp}_{K}\left(R_{e}\right)$, and, by (2), $\operatorname{Comp}_{K}(\tilde{R})=\operatorname{Comp}_{K}\left(P_{a}\right)$. Similarly, one can deduce that $\operatorname{Comp}_{K}(\tilde{R}) \neq \operatorname{Comp}_{K}\left(Q_{c}\right)$. Thus, by (3), $\operatorname{Comp}_{K}(\tilde{Q})=\operatorname{Comp}_{K}\left(R_{e}\right)$, and, again, we can deduce that $\operatorname{Comp}_{K}(\tilde{Q}) \neq \operatorname{Comp}_{K}\left(P_{a}\right)$. As $P, Q$, and $R$ are extreme-joined, we conclude that

$$
\begin{aligned}
& \operatorname{Comp}_{K}\left(P_{a}\right)=\operatorname{Comp}_{K}\left(P_{b}\right) \neq \operatorname{Comp}_{K}(\tilde{Q}), \\
& \operatorname{Comp}_{K}\left(Q_{c}\right)=\operatorname{Comp}_{K}\left(Q_{d}\right) \neq \operatorname{Comp}_{K}(\tilde{R}), \\
& \operatorname{Comp}_{K}\left(R_{e}\right)=\operatorname{Comp}_{K}\left(R_{f}\right) \neq \operatorname{Comp}_{K}(\tilde{P}) .
\end{aligned}
$$

See Figure 2(b).

Hence, by (5), (6), and (7), we have three paths, $P_{a} \cdot a c \cdot \widetilde{Q} \cdot d b \cdot P_{b}, Q_{c} \cdot c e \cdot \widetilde{R} \cdot f d \cdot Q_{d}$, and $R_{e} \cdot e a \cdot \widetilde{P} \cdot b f \cdot R_{f}$, whose lengths sum more than $3 L$, which leads to a contradiction.

The previous lemma examines how longest paths that are extreme-joined by a clique behave. The following lemma examines the case in which the longest paths are extreme-separated. Observe that, in both cases, we are only considering longest paths that cross the clique and touch it at most twice. 

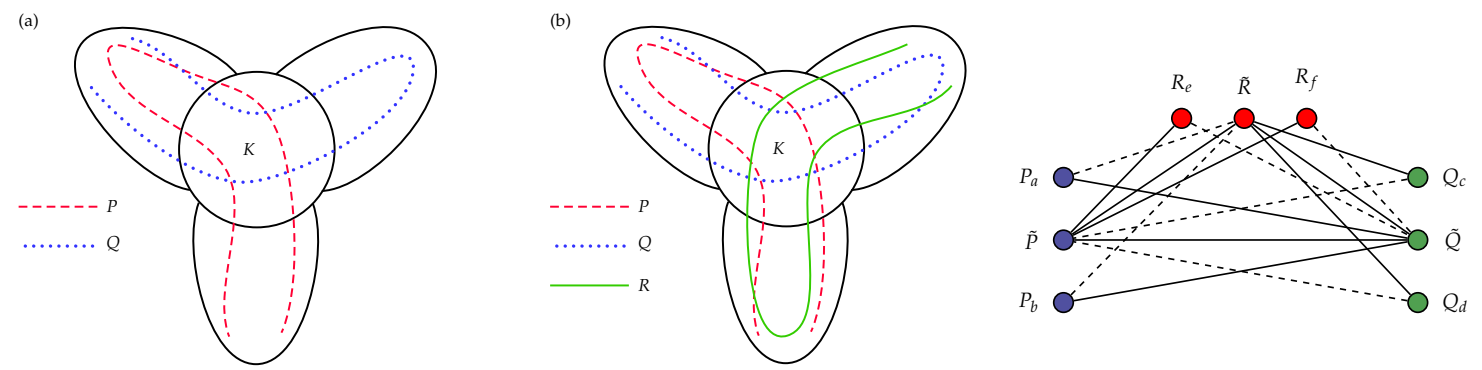

Figure 2: (a) Paths $P$ and $Q$ from the proof of Lemma 12 (b) Paths $P, Q$, and $R$, and a tripartite graph representing the interaction between their parts. The vertex set of the graph has three vertices for each of the paths, one for each part. The edge set contains two types of edges: the straight edges connect parts that are componentdisjoint and the dashed edges connect parts that are not component-disjoint. When the interaction between two parts is not determined, we omit the edge between them.

Lemma 13. Let $G$ be a graph with a clique $K$ and let $\mathscr{C}$ be the set of all longest paths in $G$ that are extreme-separated and touch $K$ at most twice. Every two elements of $\mathscr{C}$ have a common vertex of $K$.

Proof. Let $P$ and $Q$ be two arbitrary paths in $\mathscr{C}$. Suppose by contradiction that $P \cap Q \cap K=\varnothing$. As $P$ is extreme-separated by $K$, path $P$ crosses $K$ and therefore $P$ either 1-touches or 2-touches $K$. To address these two possibilities at once, let $x$ and $y$ be such that $P$ touches $K$ at $x$ and $y$, with $x \neq y$ if $P$ 2-touches $K$. Also, if $x=y$, then let $P_{x}$ and $P_{y}$ be different $x$-tails of $P$ and let $\widetilde{P}$ be the path consisting of only the vertex $x$. Let $w$ and $z$, and possibly $Q_{w}, Q_{z}$, and $\widetilde{Q}$, be defined similarly for $Q$.

As both $P$ and $Q$ are extreme-separated by $K$, the tail $P_{x}$ is component-disjoint from at least one in $\left\{Q_{w}, Q_{z}\right\}$. Analogously, $P_{y}$ is component-disjoint from at least one in $\left\{Q_{w}, Q_{z}\right\}, Q_{w}$ is component-disjoint from at least one in $\left\{P_{x}, P_{y}\right\}$ and $Q_{z}$ is component-disjoint from at least one in $\left\{P_{x}, P_{y}\right\}$. We may assume without loss of generality that $P_{x}$ and $Q_{w}$ are component-disjoint and that $P_{y}$ and $Q_{z}$ are component-disjoint. (Otherwise interchange $w$ and $z$.) Observe also that $\widetilde{P}$ is component-disjoint from at least one in $\left\{Q_{w}, Q_{z}\right\}$. Without loss of generality, assume that $\widetilde{P}$ is component-disjoint from $Q_{w}$. (Otherwise interchange $x$ and $y$, and $w$ and $z$ simultaneously.)

Note that $\widetilde{Q}$ is component-disjoint from at least one in $\left\{P_{x}, P_{y}\right\}$. First suppose that $\widetilde{Q}$ is component-disjoint from $P_{y}$. (See a representation of the interactions between the parts of $P$ and $Q$ in Figure $3(\mathrm{a})$.) Then, one of the paths $P_{x} \cdot \widetilde{P} \cdot y w \cdot Q_{w}$ or $P_{y} \cdot y w \cdot \widetilde{Q} \cdot Q_{z}$ is longer than $L$, a contradiction. Now suppose that $\widetilde{Q}$ is not component-disjoint from $P_{y}$, that is, $\operatorname{Comp}_{K}(\widetilde{Q})=\operatorname{Comp}_{K}\left(P_{y}\right)$, and thus $\widetilde{Q}$ is component-disjoint from $P_{x}$. If $\widetilde{P}$ and $\widetilde{Q}$ are componentdisjoint (see Figure $3(\mathrm{~b})$ ), then one of $P_{x} \cdot \widetilde{P} \cdot y z \cdot \widetilde{Q} \cdot Q_{w}$ or $P_{y} \cdot y z \cdot Q_{z}$ is longer than $L$, a contradiction. If $\widetilde{P}$ and $\widetilde{Q}$ are not component-disjoint, that is, $\operatorname{Comp}_{K}(\widetilde{P})=\operatorname{Comp}_{K}(\widetilde{Q})$, then, as $\operatorname{Comp}_{K}(\widetilde{Q})=\operatorname{Comp}_{K}\left(P_{y}\right)$ and $P_{y}$ and $Q_{z}$ are component-disjoint, we have that $Q_{z}$ is componentdisjoint from $\widetilde{P}$ (see Figure $3(\mathrm{c})$ ). Thus, one of the paths $P_{x} \cdot x z \cdot \widetilde{Q} \cdot Q_{w}$ or $P_{y} \cdot \widetilde{P} \cdot x z \cdot Q_{z}$ is longer than $L$, a contradiction.

The following lemma synthesizes the two previous lemmas. It says that, for every clique, when the transversal is not in it, we would have a longest path that is fenced by the clique. Observe that the lemma is valid only for chordal graphs. Remember that $\omega(G)$ is the size of a maximum clique in $G$. A $k$-clique is a subset of $k$ vertices in $G$ that are pairwise adjacent.

Lemma 14. Let $G$ be a connected chordal graph with a $k$-clique $K$. One of the following is true: 


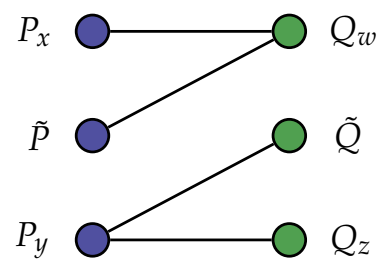

(a)

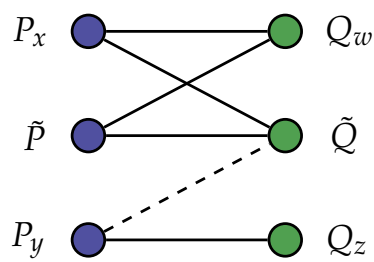

(b)

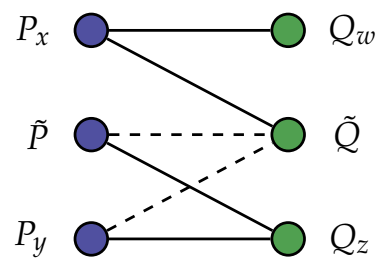

(c)

Figure 3: Each bipartite graph represents the situation of the paths $P$ and $Q$ in one of the cases of the proof of Lemma13 Each side of the bipartition has three vertices that represent the parts of each path. There is a straight edge in the graph if the two corresponding vertices are component-disjoint and a dashed edge if they are not.

(a) $\operatorname{lpt}(G) \leq \max \{1, \omega(G)-2\}$.

(b) There exists a longest path that does not touch $\mathrm{K}$.

(c) There exists a vertex $v$ of $K$ such that there is a longest path that is fenced by $K$ and 1-touches $K$ at $v$. Moreover, no longest path that 1-touches $K$ at $v$ crosses $K$.

(d) There exists an edge e of $K$ such that there is a longest path that is fenced by $K$ and 2-touches $K$ at the ends of $e$. Moreover, no longest path that 2-touches $K$ at the ends of e crosses $K$.

Proof. We will prove that the negation of $(a),(b)$, and $(c)$ implies $(d)$. So, suppose that no clique of size $\max \{1, \omega(G)-2\}$ is a longest path transversal in $G$, that every longest path touches $K$ at least once, and that, if a vertex $v$ is such that some longest path 1-touches $K$ at $v$, then there exists a longest path that 1-touches $K$ at $v$ and crosses $K$. If $k \leq \max \{1, \omega(G)-2\}$, then either $(a)$ or $(b)$ holds. So we may assume that $k \geq \max \{1, \omega(G)-2\}+1$. If $\omega(G) \leq 3$, then $\operatorname{tw}(G) \leq 2$ by Proposition 3 , and $(a)$ holds by Chen et al. [6]. We conclude that $\omega(G) \geq 4$ and $k \in\{\omega(G)-1, \omega(G)\}$.

Case 1. There is a longest path that 1-touches $K$.

If $k=\omega(G)-1$ then, as $(a)$ and $(b)$ do not hold, for every vertex in $K$, there exists a longest path that 1-touches $K$ at that vertex. Also, as $(c)$ is false, we may assume that each such path crosses $K$, a contradiction to Lemma 13, because $k \geq 3$. So $k=\omega(G)$. As (c) does not hold, and we are assuming that there is a longest path that 1-touches $K$, there exists a longest path $P$ that 1-touches $K$ at a vertex $v$ and crosses $K$. As $(a)$ does not apply, for every $(k-2)$-clique in $K$ containing $v$, there exists a longest path that does not contain any vertex in that clique. If any of these longest paths 1-touches $K$ at a vertex $w$, then, as $(c)$ does not hold, there is a longest path that crosses $K$ at $w$, contradicting Lemma 13 . Hence, for every edge in $K$ not incident to $v$, there exists a longest path that 2-touches $K$ at the ends of that edge. Again, by Lemma 13, as $P$ crosses $K$ at $v$, none of these paths is extreme-separated by $K$. As $k \geq 4$, there are at least three such paths. By Lemma 12, one of these edges, call it $e$, is such that no longest path crosses $K$ and 2 -touches $K$ at the ends of $e$. Moreover, we know that there is a longest path that 2-touches $K$ at the ends of $e$ and, by the previous discussion, that path is fenced by $K$. So $(d)$ holds.

Case 2. Every longest path touches $K$ at least twice.

If $k=\omega(G)-1$, then any subset of vertices of $K$ of size $\omega(G)-2$ is a longest path transversal, and $(a)$ would hold. Thus we may also assume that $k=\omega(G)$. As $\operatorname{lpt}(G)>\omega(G)-2=k-2$, for every edge of $K$, there exists a longest path that 2-touches $K$ at the ends of that edge. As $k \geq 4$, 
there are at least six nonequivalent longest paths that 2-touch $K$. Suppose by contradiction that (d) does not hold. Hence, we may assume that these six paths cross $K$. By Lemma 12, four of these longest paths are extreme-separated by $K$. As at least two of the corresponding four edges of $K$ are disjoint, by Lemma 13, we have a contradiction.

We can finally prove our main result.

Theorem 15. For every connected chordal graph $G, \operatorname{lpt}(G) \leq \max \{1, \omega(G)-2\}$.

Proof. Suppose by contradiction that $\operatorname{lpt}(G)>\max \{1, \omega(G)-2\}$. Then, for every clique $K$ in $G$, there exists a longest path fenced by $K$ as in $(b),(c)$, or $(d)$ of Lemma 14 We create a directed graph $D$, that admits antiparalell arcs, as follows. Let $(T, \mathcal{V})$ be a tree decomposition of $G$. The nodes of $D$ are exactly the nodes of $T$. Let $t$ be a node in $T$ and let $P$ be a longest path in $G$ fenced by $V_{t}$ that satisfies one of the conditions $(b),(c)$, or $(d)$ of Lemma 14 By Proposition 7 , there exists a neighbor $t^{\prime}$ of $t$ in $T$ such that $\operatorname{Branch}_{t}(P)=\operatorname{Branch}_{t}\left(t^{\prime}\right)$. Hence $t t^{\prime}$ is an arc in $D$. Thus every node of $D$ is the tail of at least one arc in $D$.

Let $t t^{\prime}$ be the last arc of a maximal directed path in $D$. As $T$ is a tree, $t^{\prime} t$ is also an arc in $D$, which implies that there exist two longest paths $P$ and $Q$ in $G$ such that $\operatorname{Branch}_{t}(P)=\operatorname{Branch}_{t}\left(t^{\prime}\right)$ and $\operatorname{Branch}_{t^{\prime}}(Q)=\operatorname{Branch}_{t^{\prime}}(t)$, where $P$ is fenced by $V_{t}$ and $Q$ is fenced by $V_{t^{\prime}}$, and both satisfy one of the conditions $(b),(c)$, or $(d)$ of Lemma 14

From now on, we assume that $(T, \mathcal{V})$ is a tree decomposition of $G$ as in Proposition 2 Note that the bags containing vertices of $P$ are only in $\operatorname{Branch}_{t}\left(t^{\prime}\right) \cup\{t\}$, and the bags containing vertices of $Q$ are only in $\operatorname{Branch}_{t^{\prime}}(t) \cup\left\{t^{\prime}\right\}$. As $\operatorname{Branch}_{t}\left(t^{\prime}\right)$ and $\operatorname{Branch}_{t^{\prime}}(t)$ are disjoint, $P \cap Q \subseteq V_{t} \cup V_{t^{\prime}}$. Let $u$ be a vertex such that $u \in V_{t} \backslash V_{t^{\prime}}$. Suppose for a moment that $P$ contains $u$ and let $v$ be a neighbor of $u$ in $P$. By Proposition 9 , vertex $v$ cannot be in $\operatorname{Branch}_{t}\left(t^{\prime}\right)$, so $v \in V_{t}$. This implies that $u v$ is an edge in $V_{t}$ and, as $V_{t}$ is a clique, $P$ contains all vertices of $V_{t}$, contradicting the fact that $P$ is fenced. So $P$ does not contain vertices in $V_{t} \backslash V_{t^{\prime}}$. By a similar reasoning, $Q$ does not contain vertices in $V_{t^{\prime}} \backslash V_{t}$. Thus $P \cap Q \subseteq V_{t} \cap V_{t^{\prime}}$. As $G$ is connected,

$$
P \cap Q=P \cap Q \cap V_{t} \cap V_{t^{\prime}} \neq \varnothing .
$$

This implies that $P \cap V_{t} \neq \varnothing$ and $Q \cap V_{t^{\prime}} \neq \varnothing$, therefore none of $P$ and $Q$ satisfies condition $(b)$ of Lemma 14 .

Suppose for a moment that $\left|V_{t} \cap V_{t^{\prime}}\right| \leq \omega(G)-2$. Then, as $\operatorname{lpt}(G)>\max \{1, \omega(G)-2\}$, there exists a longest path $R$ that does not contain any vertex of $V_{t} \cap V_{t^{\prime}}$. As $G$ is connected, $R$ intersects $P$. As $P$ does not contain vertices in $V_{t} \backslash V_{t^{\prime}}$ and $R$ does not contain vertices in $V_{t} \cap V_{t^{\prime}}$, we have that $P \cap R \nsubseteq V_{t}$. As the bags containing vertices of $P$ are only in $\operatorname{Branch}_{t}\left(t^{\prime}\right) \cup\{t\}, R$ has a vertex in a bag of $\operatorname{Branch}_{t}\left(t^{\prime}\right)$. A similar reasoning, with $Q$ instead of $P$, shows that $R$ also has a vertex in a bag of $B \operatorname{ranch}_{t^{\prime}}(t)$. This is a contradiction to Proposition 8 , as $R$ contains no vertex in $V_{t} \cap V_{t^{\prime}}$. Hence $\left|V_{t} \cap V_{t^{\prime}}\right| \geq \omega(G)-1$. Moreover, as both $V_{t}$ and $V_{t^{\prime}}$ are maximal (and different), we conclude that $\left|V_{t}\right|=\left|V_{t^{\prime}}\right|=\omega(G)$ and $\left|V_{t} \cap V_{t^{\prime}}\right|=\omega(G)-1$.

Remember that none of $P$ and $Q$ satisfies condition $(b)$ of Lemma 14, So $P$ touches $V_{t}$ at least once and $Q$ touches $V_{t^{\prime}}$ at least once. First suppose that $P$ 1-touches $V_{t}$ at a vertex $v$. That is, $P$ satisfies condition (c) of Lemma 14, By (8),

$$
\varnothing \neq P \cap Q=P \cap V_{t} \cap Q \cap V_{t^{\prime}}=\{v\} \cap V_{t^{\prime}} \cap Q=\{v\} \cap Q .
$$

So $P \cap Q=\{v\}$. That is, $P$ and $Q$ only intersect each other at $v$, which implies that $v$ divides both longest paths in half. Let $P^{\prime}$ and $P^{\prime \prime}$ be the two $v$-tails of $P$, and let $Q^{\prime}$ and $Q^{\prime \prime}$ be the two $v$-tails of $Q$. Let $\{u\}=V_{t} \backslash V_{t^{\prime}}$ and $\{w\}=V_{t^{\prime}} \backslash V_{t}$. As $P$ 1-touches $V_{t}$, we may assume without loss of 
generality that $w \notin P^{\prime}$. Suppose that $Q$ also 1-touches $V_{t^{\prime}}$. Then, we may assume without loss of generality that $u \notin Q^{\prime}$. But then $P^{\prime} \cdot Q^{\prime}$ is a longest path that 1-touches $V_{t}$ at $v$ and crosses $V_{t}$. As $P$ exists, condition (c) of Lemma 14 is not satisfied, a contradiction. Now suppose that $Q$ 2-touches $V_{t^{\prime}}$ at $\{v, x\}$. Note that $Q_{v}=Q^{\prime}$ or $Q_{v}=Q^{\prime \prime}$. If $u \notin Q_{v}$ then $P^{\prime} \cdot Q_{v}$ is a longest path that 1-touches $V_{t}$ at $v$ and crosses $V_{t}$, again a contradiction. Hence, $u \in Q_{v}$. But then $P^{\prime} \cdot \tilde{Q} \cdot Q_{x}$ is a longest path that 2-touches $V_{t^{\prime}}$ and crosses $V_{t^{\prime}}$. As $Q$ exists, condition $(d)$ of Lemma 14 is not satisfied, again a contradiction. Therefore $P$ touches $V_{t}$ at least twice.

By a similar reasoning, we may conclude that $Q$ touches $V_{t^{\prime}}$ at least twice. So both $P$ and $Q$ must satisfy condition $(d)$ of Lemma 14 Suppose that $P$ 2-touches $V_{t}$ at the ends of edge $x y$. First suppose that $Q$ also 2-touches $V_{t^{\prime}}$ at the same vertices. Then, $\left|P_{x}\right|=\left|Q_{x}\right|,\left|P_{y}\right|=\left|Q_{y}\right|$, and $|\tilde{P}|=|\tilde{Q}|$. If $u \notin Q_{x}$ then $P_{y} \cdot \tilde{P} \cdot Q_{x}$ is a longest path that 2-touches $V_{t}$ and crosses $V_{t}$. As $P$ exists, condition $(d)$ of Lemma 14 is not satisfied, a contradiction. Hence, $u \in Q_{x}$ and $u \notin \tilde{Q}$. Then $P_{x} \cdot \tilde{Q} \cdot P_{y}$ is a longest path that 2-touches $V_{t}$ and crosses $V_{t}$, again a contradiction. Hence, we may assume that $Q$ 2-touches $V_{t^{\prime}}$ at the ends of an edge $y z$ with $z \neq x$. Then $P_{x} \cdot x z \cdot \tilde{Q} \cdot P_{y}$ and $Q_{z} \cdot z x \cdot \tilde{P} \cdot Q_{y}$ are paths, yielding the final contradiction.

The previous theorem implies the following results.

Corollary 16. If $G$ is a tree or a 2-tree, then $\operatorname{lpt}(G)=1$.

Corollary 17. If $G$ is a 3-tree or a connected chordal planar graph, then $\operatorname{lpt}(G) \leq 2$.

\section{BIPARTITE PERMUTATION GRAPHS}

Let $\mathcal{L}_{1}$ and $\mathcal{L}_{2}$ be two parallel lines in the plane. Consider two sets $X=\left\{x_{1}, x_{2}, \ldots, x_{n}\right\}$ and $Y=\left\{y_{1}, y_{2}, \ldots, y_{m}\right\}$ of segments that joins a point in $\mathcal{L}_{1}$ with a point in $\mathcal{L}_{2}$, such that the extremes of every two elements in $X \cup Y$ are pairwise disjoint. Moreover, every two elements in $X$ do not intersect each other and every two elements in $Y$ do not intersect each other.

Let $\sigma$ be the function that maps the extreme in $\mathcal{L}_{1}$ of a segment to the other extreme. That is, if $r_{i}$ is the extreme in $\mathcal{L}_{1}$ of a segment in $X$, then the other extreme is $\sigma\left(r_{i}\right)$; and if $s_{i}$ is the extreme in $\mathcal{L}_{1}$ of a segment in $Y$, then the other extreme is $\sigma\left(s_{i}\right)$. Consider an associated bipartite graph $G=(X, Y, E)$ where $x y \in E$ if and only if the segments $x$ and $y$ intersect each other. We call the tuple $\left(\mathcal{L}_{1}, \mathcal{L}_{2}, X \cup Y, \sigma\right)$ a line representation of $G$ and a graph is called a bipartite permutation graph if it has a line representation. (See Figure 4)

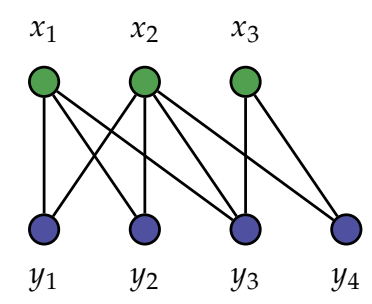

(a)

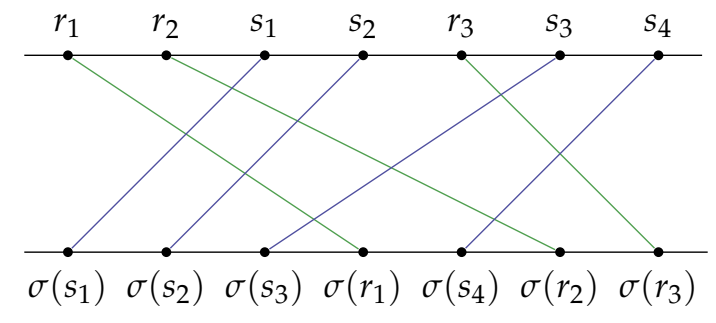

(b)

Figure 4: (a) A bipartite permutation graph. (b) Its corresponding line representation.

In what follows, we assume that $G=(X, Y, E)$ is a connected bipartite permutation graph, with a line representation $\left(\mathcal{L}_{1}, \mathcal{L}_{2}, X \cup Y, \sigma\right)$, where $X=\left\{x_{1}, x_{2}, \ldots, x_{n}\right\}$ and $Y=\left\{y_{1}, y_{2}, \ldots, y_{m}\right\}$. 
We also assume that $r_{i}$ is the extreme of $x_{i}$ in $\mathcal{L}_{1}$ and that $s_{i}$ is the extreme of $y_{i}$ in $\mathcal{L}_{1}$. Moreover, we consider that the sets $X$ and $Y$ are ordered by its extremes in $\mathcal{L}_{1}$. That is, $r_{i}<r_{j}$ if and only if $i<j$, for every $x_{i}$ and $x_{j}$ in $X$; and $s_{i}<s_{j}$ if and only if $i<j$, for every $y_{i}$ and $y_{j}$ in $Y$. For two elements $x_{i}$ and $x_{j}$ in $X$ with $i<j$, we also say that $x_{i}<x_{j}$, and we do the same for $Y$. Next we show some basic properties of bipartite permutation graphs.

Proposition 18. $\sigma\left(r_{1}\right)<\sigma\left(r_{2}\right)<\cdots<\sigma\left(r_{n}\right)$ and $\sigma\left(s_{1}\right)<\sigma\left(s_{2}\right)<\cdots<\sigma\left(s_{m}\right)$.

Proof. Suppose by contradiction that there exist $i$ and $j$ such that $r_{i}<r_{j}$ and $\sigma\left(r_{i}\right)>\sigma\left(r_{j}\right)$. Hence $x_{i}$ and $x_{j}$ would be adjacent, a contradiction to the fact that $G$ is bipartite. A similar proof applies for $Y$ instead of $X$.

The next proposition says that the neighborhood of a vertex is either completely to its left or completely to its right.

Proposition 19. If $x_{i}, x_{j} \in N\left(y_{k}\right)$ for some $i, j, k$, then $\left(r_{i}-s_{k}\right)\left(r_{j}-s_{k}\right)>0$. In a similar way, if $y_{i}, y_{j} \in N\left(x_{k}\right)$ for some $i, j, k$, then $\left(s_{i}-r_{k}\right)\left(s_{j}-r_{k}\right)>0$.

Proof. Suppose by contradiction that there exist $i, j, k$ such that $r_{i}<s_{k}<r_{j}$ and $r_{i}, r_{j} \in N\left(s_{k}\right)$. Then $\sigma\left(r_{j}\right)<\sigma\left(s_{k}\right)<\sigma\left(r_{i}\right)$, a contradiction to Proposition 18 A similar proof applies when considering $Y$ instead of $X$.

Proposition 20. If $x_{i}$ is adjacent to $y_{j_{1}}$ and $y_{j_{2}}$, with $j_{1} \leq j_{2}$, then $x_{i}$ is adjacent to every $y_{j}$ with $j_{1} \leq$ $j \leq j_{2}$. If $x_{i_{1}}$ and $x_{i_{2}}$, with $i_{1} \leq i_{2}$, are adjacent to $y_{j}$, then $y_{j}$ is adjacent to every $x_{i}$ with $i_{1} \leq i \leq i_{2}$.

Proof. The two statements are symmetric, so we only analyze the first one. The case in which $j \in\left\{j_{1}, j_{2}\right\}$ is clear, so we may assume that $j_{1}<j<j_{2}$. By Proposition 19, either $x_{i}<y_{j_{1}}<y_{j}<y_{j_{2}}$ or $y_{j_{1}}<y_{j}<y_{j_{2}}<x_{i}$. Consider the first case. As $x_{i} y_{j_{2}} \in E$, we have that $\sigma\left(s_{j_{2}}\right)<\sigma\left(r_{i}\right)$. Also, $\sigma\left(s_{j}\right)<\sigma\left(s_{j_{2}}\right)$ by Proposition 18, So $\sigma\left(s_{j}\right)<\sigma\left(r_{i}\right)$, implying that $x_{i}$ and $y_{j}$ are adjacent. We can apply a similar argument to deduce that $y_{j}$ and $x_{i}$ are also adjacent in the second case.

The following two properties are very important, as they will be used repeatedly throughout the next proofs.

Proposition 21. If $x_{i_{1}} y_{j_{2}}, x_{i_{2}} y_{j_{1}} \in E$, with $i_{1} \leq i_{2}$ and $j_{1} \leq j_{2}$, then $x_{i_{1}} y_{j_{1}}, x_{i_{2}} y_{j_{2}} \in E$. In other words, $\left\{x_{i_{1}}, x_{i_{2}}, y_{j_{1}}, y_{j_{2}}\right\}$ induces a complete bipartite graph.

Proof. The case in which $j_{1}=j_{2}$ or $i_{1}=i_{2}$ is clear, so let us assume that $i_{1}<i_{2}$ and $j_{1}<j_{2}$. First suppose that $r_{i_{1}}<s_{j_{2}}$. Then $\sigma\left(s_{j_{2}}\right)<\sigma\left(r_{i_{1}}\right)$ because $x_{i_{1}} y_{j_{2}} \in E$. By Proposition 18, we have that $\sigma\left(r_{i_{1}}\right)<\sigma\left(r_{i_{2}}\right)$ and $\sigma\left(s_{j_{1}}\right)<\sigma\left(s_{j_{2}}\right)$. So

$$
\sigma\left(s_{j_{1}}\right)<\sigma\left(s_{j_{2}}\right)<\sigma\left(r_{i_{1}}\right)<\sigma\left(r_{i_{2}}\right) .
$$

Hence, as $x_{i_{2}} y_{j_{1}} \in E$, we have that $r_{i_{2}}<s_{j_{1}}$, and

$$
r_{i_{1}}<r_{i_{2}}<s_{j_{1}}<s_{j_{2}} .
$$

By (9) and (10), we derive that $x_{i_{1}} y_{j_{1}}, x_{i_{2}} y_{j_{2}} \in E$. (See Figure $5(\mathrm{a})$.)

Now suppose that $r_{i_{1}}>s_{j_{2}}$. Then

$$
s_{j_{1}}<s_{j_{2}}<r_{i_{1}}<r_{i_{2}} .
$$

As $x_{i_{2}} y_{j_{1}} \in E$, we have that $\sigma\left(r_{i_{2}}\right)<\sigma\left(s_{j_{1}}\right)$. Using Proposition 18, we deduce that

$$
\sigma\left(r_{i_{1}}\right)<\sigma\left(r_{i_{2}}\right)<\sigma\left(s_{j_{1}}\right)<\sigma\left(s_{j_{2}}\right) .
$$

By (11) and (12), we derive that $x_{i_{1}} y_{j_{1}}, x_{i_{2}} y_{j_{2}} \in E$. (See Figure $5(b)$.) 


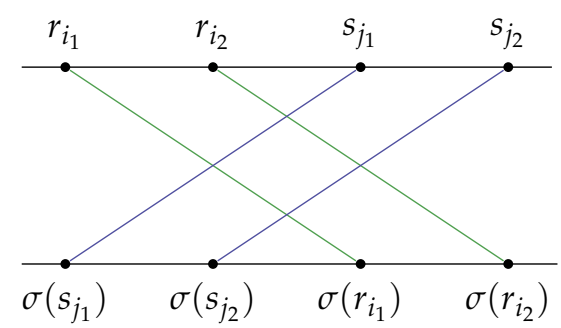

(a)

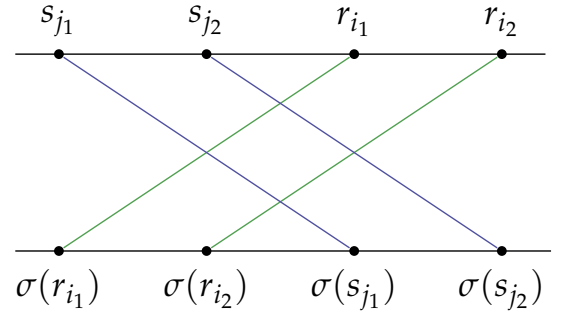

(b)

Figure 5: The two cases in the proof of Proposition 21]

Proposition 22. Let $x_{i_{1}} \leq x_{i_{2}} \leq x_{i_{3}} \leq x_{i_{4}}$ be vertices in $X$ and $y_{j_{1}} \leq y_{j_{2}} \leq y_{j_{3}} \leq y_{j_{4}}$ be vertices in $Y$. If $x_{i_{1}} y_{j_{4}}$ and $x_{i_{4}} y_{j_{1}}$ are in $E$, then $x_{i_{2}} y_{j_{3}}, y_{j_{2}} x_{i_{3}}, x_{i_{2}} y_{j_{2}}$, and $x_{i_{3}} y_{j_{3}}$ are in $E$. In other words, $\left\{x_{i_{2}}, x_{i_{3}}, y_{j_{2}}, y_{j_{3}}\right\}$ induces a complete bipartite graph.

Proof. By Proposition 21, we have that $x_{i_{1}} y_{j_{1}}$ and $x_{i_{4}} y_{j_{4}}$ are in E. By applying Proposition 20, once for $x_{i_{1}}$ and once for $x_{i_{4}}$, we deduce that $x_{i_{1}} y_{j_{2}}, x_{i_{1}} y_{j_{3}}, x_{i_{4}} y_{j_{2}}, x_{i_{4}} y_{j_{3}}$ are in $E$, and now applying it for $y_{j_{2}}$ and for $y_{j_{3}}$, we deduce that $x_{i_{2}} y_{j_{2}}, x_{i_{3}} y_{j_{2}}, x_{i_{2}} y_{j_{3}}$ and $x_{i_{3}} y_{j_{3}}$ are in $E$.

Until now, we have used the line representation of $G$ to prove some properties. From now on, we will not need this line representation anymore. That is, we only need to concentrate in the graph $G$, viewed as a bipartite graph that has the previous properties.

We are interested in how do longest paths behave in a bipartite permutation graph. We begin by showing that every longest path can be converted into another longest path with the same set of vertices that is ordered in some way. As we only care about vertex intersection of longest paths, we will be only interested in such ordered paths. To be more precise, if $P=a_{1} b_{1} a_{2} b_{2} \cdots a_{k} b_{k}$ is a path in $G$, we say that $P$ is ordered if $a_{1}<a_{2}<\cdots<a_{k}$ and $b_{1}<b_{2}<\cdots<b_{k}$. A similar definition applies when $P$ has even length.

Let $P$ be a path in $G$ with $P \cap X=\left\{a_{1}, a_{2}, \ldots, a_{|P \cap X|}\right\}$ and $P \cap Y=\left\{b_{1}, b_{2}, \ldots, b_{|P \cap Y|}\right\}$, so that $a_{1}<a_{2}<\cdots<a_{|P \cap X|}$ and $b_{1}<b_{2}<\cdots<b_{|P \cap Y|}$. For every $i \in\{1, \ldots,|P \cap X|\}$, let $X_{i}=\left\{a_{1}, a_{2}, \ldots, a_{i}\right\}$ and $\bar{X}_{i}=P \cap\left(X \backslash X_{i}\right)$. For every $i \in\{1, \ldots,|P \cap Y|\}$, let $Y_{i}=\left\{b_{1}, b_{2}, \ldots, b_{i}\right\}$ and $\bar{Y}_{i}=P \cap\left(Y \backslash Y_{i}\right)$. We denote by $d_{P}\left(X_{i}\right)$ the sum $\sum_{v \in X_{i}} d_{P}(v)$ and by $d_{P}\left(Y_{j}\right)$ the sum $\sum_{w \in Y_{j}} d_{P}(w)$.

Proposition 23. Let $i, j$ be such that $1 \leq i \leq|P \cap X|, 1 \leq j \leq|P \cap Y|$, and either $i<|P \cap X|$ or $j<|P \cap Y|$. Then, there exists either an edge in $P$ from $X_{i}$ to $\bar{Y}_{j}$, or an edge in $P$ from $Y_{j}$ to $\bar{X}_{i}$.

Proof. Without loss of generality, assume that $i<|P \cap X|$. Let $v \in P \cap\left(X \backslash X_{i}\right)$. Suppose by contradiction that no such edge exists. Then, there is no path, in the subgraph of $G$ induced by $E(P)$, between $v$ and a vertex in $X_{i} \cup Y_{j}$, a contradiction.

Proposition 24. Let $i, j$ be such that $1 \leq i \leq|P \cap X|, 1 \leq j \leq|P \cap Y|$, and either $i<|P \cap X|$ or $j<|P \cap Y|$. If $d_{P}\left(X_{i}\right) \geq d_{P}\left(Y_{j}\right)$, then there exists an edge from $X_{i}$ to $\bar{Y}_{j}$. If $d_{P}\left(Y_{j}\right) \geq d_{P}\left(X_{i}\right)$, then there exists an edge from $Y_{j}$ to $\bar{X}_{i}$.

Proof. We will prove only the first affirmation, as the proof for the second one is analogous. Suppose by contradiction that $d_{P}\left(X_{i}\right) \geq d_{P}\left(Y_{j}\right)$ and there exists no edge from $X_{i}$ to $\bar{Y}_{j}$. By 
Proposition 23, there exists at least one edge from $Y_{j}$ to $\bar{X}_{i}$, so

$$
\begin{aligned}
d_{P}\left(Y_{j}\right) & =\left\{w v \in E(P): w \in Y_{j}, v \in X_{i}\right\}+\left\{w v \in E(P): w \in Y_{j}, v \in \bar{X}_{i}\right\} \\
& =\left\{v w \in E(P): v \in X_{i}, w \in V(P)\right\}+\left\{w v \in E(P): w \in Y_{j}, v \in \bar{X}_{i}\right\} \\
& =d_{P}\left(X_{i}\right)+\left\{w v \in E(P): w \in Y_{j}, v \in \bar{X}_{i}\right\} \\
& >d_{P}\left(X_{i}\right)
\end{aligned}
$$

a contradiction.

Lemma 25. For every path $P$ in a bipartite permutation graph, there exists an ordered path with the same vertex set as $P$.

Proof. Suppose that $P \cap X=\left\{a_{1}, a_{2}, \ldots, a_{|P \cap X|}\right\}, P \cap Y=\left\{b_{1}, b_{2}, \ldots, b_{|P \cap Y|}\right\}, a_{1}<\cdots<a_{|P \cap X|}$ and $b_{1}<\cdots<b_{|P \cap Y|}$. Without loss of generality, we may assume that $|P \cap X| \geq|P \cap Y|$ and that, if $|P \cap X|=|P \cap Y|$, then $i^{*} \leq j^{*}$, where $a_{i^{*}}$ is the extreme of $P$ in $X$ and $b_{j^{*}}$ is the extreme of $P$ in $Y$. (If $i^{*}>j^{*}$, then a similar proof applies by interchanging $X$ and $Y$.) Let $k=|P \cap Y|$. We will show that

for every $i \in\{1, \ldots, k\}$, there exists an edge with one end in $X_{i}$ and the other in $\bar{Y}_{i-1}$,

and

$$
\text { for every } i \in\{1, \ldots, k-1+|P \cap X|-|P \cap Y|\} \text {, }
$$

there exists an edge with one end in $Y_{i}$ and the other in $\bar{X}_{i}$.

Proof of (13): Observe that $d_{P}(u)=1$ for at most two vertices $u$ in $X_{i}$ (the extremes of $P$ ). Therefore, $d_{P}\left(X_{i}\right) \geq 2\left|X_{i}\right|-2=2\left|Y_{i-1}\right|$. As $d_{P}(w) \leq 2$ for every $w \in Y_{i-1}$, we have that $d_{P}\left(Y_{i-1}\right) \leq 2\left|Y_{i-1}\right|$. Hence, $d_{P}\left(X_{i}\right) \geq d_{P}\left(Y_{i-1}\right)$ and, as $i-1<k$, (13) is valid by Proposition 24.

Proof of (14): First suppose that $|P \cap X|=k(=|P \cap Y|)$. As $i^{*} \leq j^{*}$, we have that $d_{P}\left(Y_{i}\right) \geq d_{P}\left(X_{i}\right)$. Indeed, if $a_{i^{*}} \in X_{i}$ then $d_{P}\left(X_{i}\right)=2\left|X_{i}\right|-2$ and $d_{P}\left(Y_{i}\right) \geq 2\left|Y_{i}\right|-2$, and if $a_{i^{*}} \notin X_{i}$ then, as $i^{*} \leq j^{*}, y_{j^{*}} \notin Y_{i}$, so $d_{P}\left(X_{i}\right)=2\left|X_{i}\right|$ and $d_{P}\left(Y_{i}\right)=2\left|Y_{i}\right|$. Thus, as $i<k$, (14) is valid by Proposition 24. Now suppose that $|P \cap X|=k+1$. Then, $d_{P}(w)=2$ for every $w \in Y_{i}$. Therefore, $d_{P}\left(Y_{i}\right)=2\left|Y_{i}\right|=2\left|X_{i}\right|$. As $d_{P}(v) \leq 2$ for every $v \in X_{i}$, we have that $d_{P}\left(X_{i}\right) \leq 2\left|X_{i}\right|$. Hence, $d_{P}\left(Y_{i}\right) \geq d_{P}\left(X_{i}\right)$ and, as $i<k+1$, (14) is valid by Proposition 24.

Let $i \in\{1, \ldots, k-1\}$. By (13), there exists a vertex $a_{q}$ in $X_{i}$ with a neighbor $b_{r^{\prime}}$ in $\bar{Y}_{i-1}$. By (14), there exists a vertex $b_{r}$ in $Y_{i}$ with a neighbor $a_{q^{\prime}}$ in $\bar{X}_{i}$. As $a_{q} \leq a_{i} \leq a_{i+1} \leq a_{q^{\prime}}$ and $b_{r} \leq b_{i} \leq b_{i} \leq b_{r^{\prime}}$, by Proposition 22, both $a_{i} b_{i}$ and $b_{i} a_{i+1}$ are edges. (See Figure 6) By (13), $a_{k} b_{k}$ is an edge, hence $a_{1} b_{1} \cdots a_{k} b_{k}$ is a path. This implies that if $|X \cap P|=k$, we are done. Also, if $|X \cap P|=k+1$, then $b_{k} a_{k+1}$ is an edge, by (14), so $a_{1} b_{1} \cdots a_{k} b_{k} a_{k+1}$ is a path.

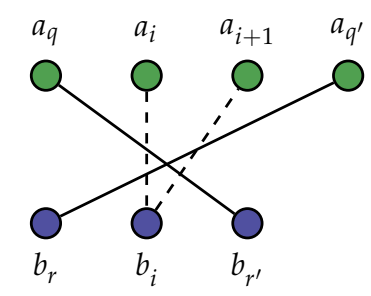

Figure 6: The proof of Lemma 25 
As observed before, Lemma 25 says that we can restrict attention to ordered longest paths from now on. Remember that we want to prove that $\operatorname{lpt}(G)=1$. We proceed in two steps. First, we will prove that $\operatorname{lpt}(G) \leq 2$. In fact, we prove that the set of ends of every edge is a longest path transversal. Finally, we will prove that one element in $\left\{x_{1}, y_{1}\right\}$ is also a longest path transversal, which implies that $\operatorname{lpt}(G)=1$.

Let $x_{i_{1}} y_{j_{1}}$ and $x_{i_{2}} y_{j_{2}}$ be two edges in $G$. We say that $x_{i_{1}} y_{j_{1}}$ and $x_{i_{2}} y_{j_{2}}$ cross each other if $\left(i_{1}-i_{2}\right)\left(j_{1}-j_{2}\right)<0$. If that is not the case, we say they are parallel. We say that $\left|i_{1}-i_{2}\right|$ is the distance in $X$ and that $\left|j_{1}-j_{2}\right|$ is the distance in $Y$ between such edges. We denote by $\operatorname{dist}_{X}\left(x_{i_{1}} y_{j_{1}}, x_{i_{2}} y_{j_{2}}\right)$ and $\operatorname{dist}_{Y}\left(x_{i_{1}} y_{j_{1}}, x_{i_{2}} y_{j_{2}}\right)$ these two values respectively.

Proposition 26. Let $P$ be a longest path and $x_{i_{1}} y_{j_{1}} \in E(P)$. Let $v w \in E(G)$. If $x_{i_{1}} y_{j_{1}}$ crosses vw, then $P$ contains at least one of $\{v, w\}$.

Proof. Suppose by contradiction that $\{v, w\} \cap V(P)=\varnothing$. Without loss of generality, suppose that $x_{i_{1}}<v$ and $y_{j_{1}}>w$. By Proposition 21, $x_{i_{1}} w$ and $v y_{j_{1}}$ are edges. Therefore, $P-x_{i_{1}} y_{j_{1}}+x_{i_{1}} w+w v+v y_{j_{1}}$ is a path longer than $P$, a contradiction.

Lemma 27. Let $G=(X, Y, E)$ be a connected bipartite permutation graph. Let $v w \in E$, with $v \in X$ and $w \in Y$. Every ordered longest path contains a vertex of $\{v, w\}$.

Proof. Suppose by contradiction that there exists an ordered longest path $P$ that does not contain either $v$ or $w$. Then, by Proposition 26, all edges of $P$ are parallel to $v w$. Let $x_{i_{1}} y_{j_{1}}$ be the edge of $P$ that is "closer" to $v w$. That is, $\operatorname{dist}_{X}\left(x_{i_{1}} y_{j_{1}}, v w\right)=\min \left\{\operatorname{dist}_{X}(e, v w): e \in E(P)\right\}$ and $\operatorname{dist}_{Y}\left(x_{i_{1}} y_{j_{1}}, v w\right)=\min \left\{\operatorname{dist}_{X}(e, v w): e \in E(P)\right\}$. Observe that, as $P$ is an ordered path, one of $\left\{x_{i_{1}}, y_{j_{1}}\right\}$ is an extreme of $P$. Suppose that $x_{i_{1}}$ is such an extreme. (A similar proof applies when this is not the case.) Without loss of generality, we may assume that $x_{i_{1}}>v$ and that $P$ is a path with minimum value of $x_{i_{1}}$ among all such paths.

Let $H$ be the subgraph of $G$ induced by the vertices $\left\{x_{i}: i \geq i_{1}\right\} \cup\left\{y_{j}: j \geq j_{1}\right\}$. As $G$ is connected and $G \neq H$, there exists an edge between $H$ and $G-V(H)$. First suppose that such an edge is between a vertex $x_{l}$ in $H$ and a vertex $y_{r}$ in $G-V(H)$. Then, by Proposition 21, $x_{i_{1}}$ is adjacent to $y_{r}$. Hence, $y_{r} x_{i_{1}} \cdot P$ is also a path, a contradiction. (See FigureZ(a).) Now suppose that there is an edge between a vertex $x_{l}$ in $G-V(H)$ and a vertex $y_{r}$ in $H$. Then, by Proposition 21, $x_{l}$ is adjacent to $y_{j_{1}}$. So $Q=P-x_{i_{1}} y_{j_{1}}+x_{l} y_{j_{1}}$ is also a longest path. As $V(Q) \backslash V(P)=\left\{x_{l}\right\}$, we have that $w \notin Q$. Observe also that $v \notin Q$. Indeed, otherwise $Q \cdot v w$ is a path longer than $P$. Hence, by Proposition 26, all edges of $Q$ are parallel to $v w$, which implies that $v<x_{l}<x_{i_{1}}$, a contradiction to the way $P$ was chosen. (See Figure $Z(b)$.)

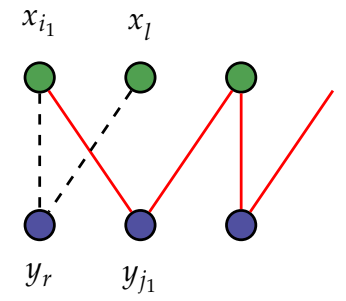

(a)

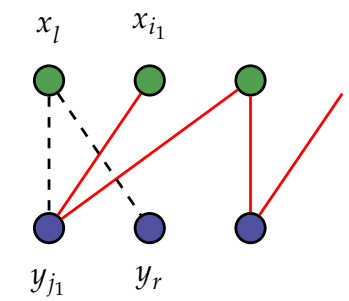

(b)

Figure 7: The two cases in the proof of Lemma 27 The solid lines represent the edges in P. 
Given a collection $\mathcal{C}$ of ordered longest paths, we say that $P \in \mathcal{C}$ is a left-most path if, for every other path $Q \in \mathcal{C}$ and for every $i$, the $i$-th vertex of $P$ in $X$ is less than or equal to the $i$-th vertex of $Q$ in $X$, and the same applies for $Y$ instead of $X$. Such a path exists because all paths in $\mathcal{C}$ are ordered.

Theorem 28. For every connected bipartite permutation graph $G=(X, Y, E), \operatorname{lpt}(G)=1$.

Proof. Suppose by contradiction that $\operatorname{lpt}(G)>1$. Then, there exists a longest path $P$ that does not contain $y_{1}$ and a longest path $Q$ that does not contain $x_{1}$. As $G$ is connected, $x_{1} y_{1}$ is an edge by Proposition 21] So, by Lemma 27, $x_{1} \in P$ and $y_{1} \in Q$. We may assume that both $P$ and $Q$ are left-most paths. Suppose without loss of generality that $n \geq m$. Thus, for all $i \in\{2, \ldots, m\}$, it suffices to prove the following conditions:

(a) $y_{i}$ is the $(2 i-3)$-th vertex of $P, x_{i-1}$ is the $(2 i-2)$-th vertex of $P, x_{i}$ is the $(2 i-3)$-th vertex of $Q$, and $y_{i-1}$ is the $(2 i-2)$-th vertex of $Q$.

(b) $x_{i} y_{i}$ is an edge.

Indeed, if that is the case, then we would have a path $R=\left(x_{1} y_{1} x_{2} y_{2} \cdots x_{m} y_{m}\right)$ of length $2 m-1$. As $P$ does not contain $y_{1}$, we would have $|P \cap Y|=\left|P \cap\left\{y_{2}, y_{3}, \ldots, y_{m}\right\}\right|=m-1$. And, as $G$ is bipartite, $|P \cap X| \leq m$. Hence $|P| \leq 2 m-2<|R|$, a contradiction, because $P$ is a longest path.

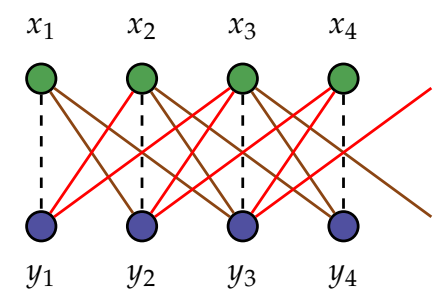

We proceed by induction on $i$. If $i=2$, we need to prove that $y_{2} x_{1}$ and $x_{2} y_{1}$ are the first edges of $P$ and $Q$ respectively, and that $x_{1} y_{1}, x_{2} y_{2}$ are edges. Remember that $x_{1} \in P$. Obviously, $x_{1}$ is not an extreme of $P$. So, as $P$ is an ordered longest path, $x_{1}$ is the second vertex of $P$. Now we will prove that $y_{2}$ is the first vertex of $P$. If $P$ starts in $y_{j}$ with $j>2$ then, as $y_{j} x_{1}$ and $x_{1} y_{1}$ are edges, $x_{1} y_{2}$ is an edge by Proposition 20. Thus $P-x_{1} y_{j}+x_{1} y_{2}$ is also a longest path, contradicting the choice of $P$. A similar reasoning shows that $x_{2} y_{1}$ is the first edge of $Q$. This implies, by Proposition 21, that $x_{2} y_{2}$ is an edge, finishing the base case of the induction.

Now fix an $i>2$ and assume that both (a) and (b) are valid for all $j<i$. Then, by the induction hypothesis, $y_{i-1} x_{i-2}$ is the $(2 i-5)$-th edge of $P$. First, we will prove that $x_{i-1}$ is the $(2 i-2)$-th vertex of $P$. Indeed, suppose that $x_{j}$ is the $(2 i-2)$-th vertex of $P$ with $j>i-1$. Let $P=P^{\prime} \cdot P^{\prime \prime}$, where $P^{\prime} \cap P^{\prime \prime}=\left\{x_{i-2}\right\}$. Then $y_{1} x_{1} y_{2} x_{2} \cdots y_{i-2} x_{i-2} \cdot P^{\prime \prime}$ is an ordered longest path that does not contain any vertex of $\left\{x_{i-1}, y_{i-1}\right\}$, a contradiction to Lemma 27 So $x_{i-1}$ is the (2i-2)-th vertex of $P$. Now, we will prove that $y_{i}$ is the $(2 i-3)$-th vertex of $P$. Suppose that $y_{j}$ is the $(2 i-3)$-th vertex of $P$ with $j>i$. Then, by Proposition 22, as, by the induction hypothesis, $x_{i-1} y_{i-1}$ is an edge, $x_{i-2} y_{i}$ and $x_{i-1} y_{i}$ are edges. Now, $P-x_{i-2} y_{j} x_{i-1}+x_{i-2} y_{i} x_{i-1}$ is also a longest path, contradicting the choice of $P$. A similar argument shows that $x_{i}$ is the $(2 i-3)$-th vertex of $Q$ and that $y_{i-1}$ is the $(2 i-2)$-th vertex of $Q$. This implies, by Proposition 21, that $x_{i} y_{i}$ is an edge, finishing the proof. 


\section{GRAPHS OF BOUNDED TREEWIDTH AND PLANAR GRAPHS}

Rautenbach and Sereni [18] proved that $\operatorname{lpt}(G) \leq \operatorname{tw}(G)+1$ for every connected graph $G$. In this section, we improve their result.

Lemma 29. Let $G$ be a connected graph. Let $(T, \mathcal{V})$ be a tree decomposition of $G$. There exists a node $t \in V(T)$ such that $V_{t}$ is a longest path transversal.

Proof. Suppose by contradiction that this is not the case. Then, for every $t \in V(T)$, there exists a longest path $P$ that does not touch $V_{t}$ (hence $P$ is fenced by $V_{t}$ ). By Proposition 7 there exists a neighbor $t^{\prime}$ of $t$ in $T$ such that $\operatorname{Branch}_{t}(P)=\operatorname{Branch}_{t}\left(t^{\prime}\right)$. We create a directed graph $D$, that admits antiparalell arcs, as follows. The nodes of $D$ are exactly the nodes of $T$. Given a node $t$ and a neighbor $t^{\prime}$ of $t$ as before, we add $t t^{\prime}$ as an arc in $D$. Note that every node of $D$ is the tail of some arc in $D$. Let $t t^{\prime}$ be the last arc of a maximal directed path in $D$. As $T$ is a tree, $t^{\prime} t$ is also an arc in $D$, which implies that there exist two longest paths $P$ and $Q$ in $G$ such that $\operatorname{Branch}_{t}(P)=\operatorname{Branch}_{t}\left(t^{\prime}\right)$ and $\operatorname{Branch}_{t^{\prime}}(Q)=\operatorname{Branch}_{t^{\prime}}(t)$, where $P$ is fenced by $V_{t}$ and $Q$ is fenced by $V_{t^{\prime}}, P$ does not touch $V_{t}$ and $Q$ does not touch $V_{t^{\prime}}$. But then, as the bags containing vertices of $P$ are only in Branch $_{t}\left(t^{\prime}\right) \cup\{t\}$, and the bags containing vertices of $Q$ are only in Branch $_{t^{\prime}}(t) \cup\left\{t^{\prime}\right\}$, the paths $P$ and $Q$ do not intersect, a contradiction.

Theorem 30. For every connected graph $G$ with treewidth $k, \operatorname{lpt}(G) \leq k$.

Proof. Let $(T, \mathcal{V})$ be a tree decomposition of $G$ as in Proposition 1 . By Lemma 29, there exists a node $t$ in $T$ such that $V_{t}$, of size $k+1$, is a longest path transversal. Suppose by contradiction that $\operatorname{lpt}(G)>k$. Then no set of $k$ vertices in $V_{t}$ is a longest path transversal. As every longest path touches $V_{t}$ at least once, for every vertex in $V_{t}$, there exists a longest path that 1-touches $V_{t}$ at that vertex. Let $P$ be a longest path that touches $V_{t}$ at $x$, let $P^{\prime}$ and $P^{\prime \prime}$ be the two $x$-tails of $P$. We will show that

$$
\operatorname{Branch}_{t}\left(P^{\prime}\right) \neq \operatorname{Branch}_{t}\left(P^{\prime \prime}\right) \text {. }
$$

Proof of (15): Suppose by contradiction that $\operatorname{Branch}_{t}\left(P^{\prime}\right)=\operatorname{Branch}_{t}\left(P^{\prime \prime}\right)=\operatorname{Branch}_{t}\left(t^{\prime}\right)$. By Proposition 1 , there exists a vertex $y$ in $V_{t} \backslash V_{t^{\prime}}$. Let $Q$ be a longest path that 1-touches $V_{t}$ at $y$. Let $Q^{\prime}$ and $Q^{\prime \prime}$ be the two $y$-tails of $P$. By Proposition 11, both $P \cap Q^{\prime}$ and $P \cap Q^{\prime \prime}$ are empty, a contradiction.

By (15), there exist two different nodes $t^{\prime}$ and $t^{\prime \prime}$ that are adjacent to $t$ in $T$ such that $t^{\prime}$ is in $\operatorname{Branch}_{t}\left(P^{\prime}\right)$ and $t^{\prime \prime}$ is in $\operatorname{Branch}_{t}\left(P^{\prime \prime}\right)$. By Proposition 11 there exists a vertex $a$ in $V_{t} \backslash V_{t^{\prime}}$ and a vertex $b$ in $V_{t} \backslash V_{t^{\prime \prime}}$. As $t^{\prime} \neq t^{\prime \prime}$, we have that $V_{t^{\prime}} \neq V_{t^{\prime \prime}}$ and $a \neq b$. Let $Q$ and $R$ be corresponding longest paths that 1-touch $V_{t}$ at $a$ and $b$ respectively. By (15), both $P$ and $Q$ cross $V_{t}$. Observe that $x \in V_{t^{\prime}} \cap V_{t^{\prime \prime}}$ by Proposition 10, and hence $a \neq x \neq b$.

Let $Q^{\prime}$ and $Q^{\prime \prime}$ be the two $a$-tails of $Q$, and let $R^{\prime}$ and $R^{\prime \prime}$ be the two $b$-tails of $R$. By Proposition 11, paths $P^{\prime}$ and $Q$ do not intersect. So, as $G$ is connected, $P^{\prime \prime}$ intersects $Q$. Since $P^{\prime \prime} \cap Q \cap V_{t}=\varnothing$, we may assume, without loss of generality, that $Q^{\prime \prime}$ intersects $P^{\prime \prime}$, thus $\operatorname{Branch}_{t}\left(Q^{\prime \prime}\right)=\operatorname{Branch}_{t}\left(P^{\prime \prime}\right)=\operatorname{Branch}_{t}\left(t^{\prime \prime}\right)$. Analogously, with a similar analysis with $R$ instead of $Q$, we may assume that $\operatorname{Branch}_{t}\left(R^{\prime}\right)=\operatorname{Branch}_{t}\left(P^{\prime}\right)=\operatorname{Branch}_{t}\left(t^{\prime}\right)$. Applying (15) with $Q$ and $R$ instead of $P$, one can show that $\operatorname{Branch}_{t}\left(Q^{\prime}\right) \neq \operatorname{Branch}_{t}\left(Q^{\prime \prime}\right)$ and that $\operatorname{Branch}_{t}\left(R^{\prime}\right) \neq \operatorname{Branch}_{t}\left(R^{\prime \prime}\right)$. Thus, $Q^{\prime}$ is disjoint from $P$, and $R^{\prime \prime}$ is disjoint from $P$. Also, as $\operatorname{Branch}_{t}\left(R^{\prime}\right)=\operatorname{Branch}_{t}\left(t^{\prime}\right)$, by Proposition 11, paths $Q$ and $R^{\prime}$ do not intersect. Analogously, $R$ and $Q^{\prime \prime}$ do not intersect.

Let $a^{\prime} \in P^{\prime \prime} \cap Q^{\prime \prime}$ be such that the subpath of $P$ with extremes $x$ and $a^{\prime}$ is internally disjoint from $Q^{\prime \prime}$. Let $Q_{1}$ and $Q_{2}$ be the two $a^{\prime}$-tails of $Q$, with $Q_{1}$ containing $a$. Let $b^{\prime} \in P^{\prime} \cap R^{\prime}$ be such that the subpath of $P$ with extremes $x$ and $b^{\prime}$ is internally disjoint from $R^{\prime}$. Let $R_{1}$ and $R_{2}$ be the 
two $b^{\prime}$-tails of $R$, with $R_{1}$ containing $b$. Let $\tilde{P}$ be the subpath of $P$ that has $a^{\prime}$ and $b^{\prime}$ as extremes. As $\tilde{P}$ is internally disjoint from both $Q$ and $R$, we have that $R_{1} \cdot \tilde{P} \cdot Q_{2}$ and $Q_{1} \cdot \tilde{P} \cdot R_{2}$ are paths whose lengths sum more than $2 L$, a contradiction.

The graph of Figure 1 has treewidth two. Hence, we have the following corollary.

Corollary 31. If $G$ is a connected partial 3-tree, then $\operatorname{lpt}(G) \in\{2,3\}$.

Planar graphs do not have bounded treewidth. However, Fomin and Thilikos [9] showed that a planar graph $G$ on $n$ vertices has treewidth at most 3.182 $\sqrt{n}$. More generally, Alon, Seymour, and Thomas [1] showed that any $K_{r}$-minor free graph on $n$ vertices has treewidth at most $r^{1.5} \sqrt{n}$. Hence, we have the following corollaries. The first of them improves the upper bound given by Rautenbach and Sereni when the graph is planar.

Corollary 32. For every connected planar graph $G$ on $n$ vertices, $\operatorname{lpt}(G) \leq 3.182 \sqrt{n}$.

Corollary 33. For every connected $K_{r}$-minor free graph $G, \operatorname{lpt}(G) \leq r^{1.5} \sqrt{n}$.

\section{FULl SUBSTAR GRAPHS}

A star is a complete bipartite graph $K_{1, k}$, for some integer $k$. If $k \geq 2$, we call the unique vertex of degree $k$ the center of the star. If $k=1$, we pick an arbitrary vertex to be the center of the star. Given a tree $T$, a subgraph of $T$ that is a star is called a substar of $T$. We say that a star in $T$ with center in $x$ is a full substar of $T$ if $k \geq d_{T}(x)-1$. A graph is a full substar graph if it is the intersection graph of a set of full substars of a tree. In the intersection model, we call $S_{x}$ the substar of the host tree associated with $x \in V(G)$. We use capital letters to refer to the vertices of the host tree of the intersection model and lowercase letters to refer to the vertices of the intersection graph. It can be seen from the definition that every full substar graph is also a chordal graph, since chordal graphs are the intersection graphs of subtrees of a tree. An example of a full substar graph can be seen in Figure 8 .
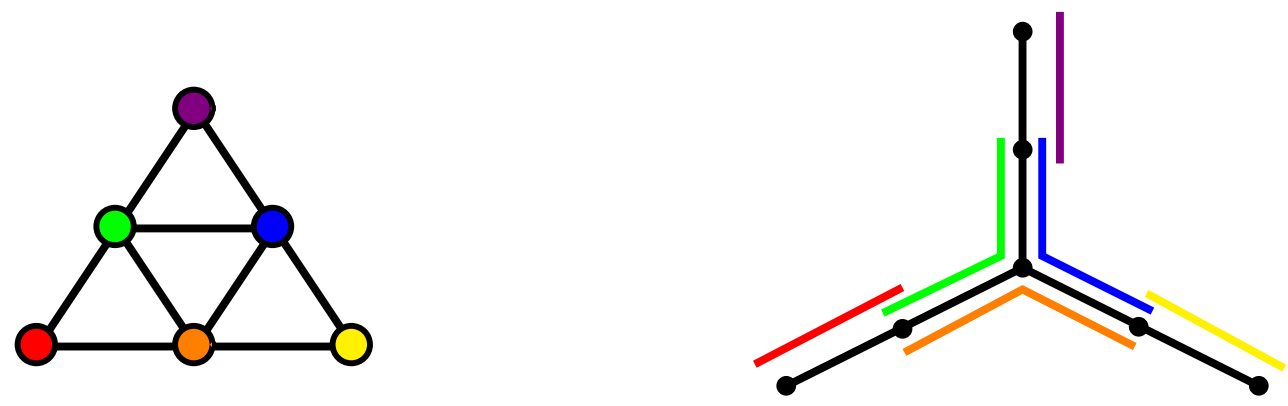

Figure 8: A full substar graph.

The definition of branch by Heinz [13] applies naturally to an arbitrary tree. Here we use it, as well as its variants introduced in Section [II. to the tree $T$. For a vertex $X \in V(T)$, let $\mathcal{C}_{X}$ be the set of vertices of $G$ whose corresponding stars are centered in $X$ and $\mathcal{C}_{Y}^{X}$ be the set of vertices of $G$ whose stars are centered in a vertex that belongs to $\operatorname{Branch}_{X}(Y)$.

In what follows, $G$ is a full substar graph and $T$ is the host tree of an intersection model for $G$.

Lemma 34. Let $x \in V(G)$ be such that $x \in \mathcal{C}_{X}$. If $P$ is a longest path in $G$ such that $x \notin V(P)$, then there exists a node $Y \in N_{T}(X)$ such that the following conditions hold: 
(i) $V(P) \subseteq \mathcal{C}_{Y}^{X} \cup \mathcal{C}_{X}$

(ii) $\left|V(P) \cap \mathcal{C}_{X}\right| \leq 1$

Moreover, if $\left|V(P) \cap \mathcal{C}_{X}\right|=1$, then $Y \notin S_{x}$.

Proof. Let $x$ and $P$ be as stated above. First suppose that $V(P) \cap \mathcal{C}_{X}=\varnothing$. Suppose by contradiction that $(i)$ is false. Then $P$ has vertices whose substars are centered in two different branches of $T$ with respect to $X$. Since $P$ contains no vertex of $\mathcal{C}_{X}$, then $P$ must contain two consecutive vertices whose stars are centered in the neighborhood of $X$ in $T$. That is, $P$ contains two consecutive vertices that are adjacent to $x$, a contradiction. This implies that $(i)$ holds in this case and, since $\left|V(P) \cap \mathcal{C}_{X}\right|=0$, (ii) also holds.

Now assume that $V(P) \cap \mathcal{C}_{X} \neq \varnothing$, and let $x^{\prime}$ be a vertex in $V(P) \cap \mathcal{C}_{X}$. Suppose by a contradiction that $(i)$ does not hold. Then $P$ has vertices whose substars are centered in two different branches of $T$ with respect to $X$. If $P$ contains two consecutive vertices whose stars are centered in different branches, we are in the previous case. Then there exists two vertices $u$ and $v$ in $P$ such that $u x^{\prime}, v x^{\prime} \in E(P)$. Since $\left|S_{x^{\prime}}\right| \geq d_{T}(X)-1$, one of $\{u, v\}$ is adjacent to $x$, a contradiction. We can conclude that $(i)$ holds in this case. Let $Y$ be as stated in $(i)$.

For showing (ii), suppose that $\left|V(P) \cap \mathcal{C}_{X}\right|>1$. Let $x^{\prime}, x^{\prime \prime} \in V(P) \cap \mathcal{C}_{X}$. If $x^{\prime} x^{\prime \prime} \in E(P)$, then we can add $x$ to $P$ between these two vertices. In this case, we may assume that $P=P_{x^{\prime}} \cdot \tilde{P} \cdot P_{x^{\prime \prime}}$, where $V\left(P_{x^{\prime}}\right) \cap V(\tilde{P})=\left\{x^{\prime}\right\}$ and $V\left(P_{x^{\prime \prime}}\right) \cap V(\tilde{P})=\left\{x^{\prime \prime}\right\}$. Observe that $x^{\prime}$ and $x^{\prime \prime}$ are not extremes of $P$, which implies that $P_{x^{\prime}}$ and $P_{x^{\prime}}$ are not empty. Thus, there exists a vertex $u$ adjacent to $x^{\prime}$ in $P_{x^{\prime}}$ and a vertex $v$ adjacent to $x^{\prime \prime}$ in $P_{x^{\prime \prime}}$. Also, there exists a vertex $w^{\prime}$ adjacent to $x^{\prime}$ in $\tilde{P}$ and a vertex $w^{\prime \prime}$ adjacent to $x^{\prime \prime}$ in $\tilde{P}$ (possibly, $w^{\prime}=w^{\prime \prime}$ ). Note that $S_{w^{\prime}} \cap S_{x^{\prime}}=\{Y\}$ and $S_{w^{\prime \prime}} \cap S_{x^{\prime \prime}}=\{Y\}$, implying that $\left\{x^{\prime}, w^{\prime}, x^{\prime \prime}, w^{\prime \prime}\right\}$ induces a clique. By a similar argument, $w^{\prime \prime}$ and $v$ are adjacent.

In this case, we can find a path longer than $P$ in $G$. Let $x_{1}$ and $x_{k}$ be the extremes of $P$ and, given $x, y \in V(P)$, let $P_{x y}$ be the subpath of $P$ that has $x$ and $y$ as its extremes. The path $P_{x_{1} x^{\prime}} \cdot x^{\prime} x \cdot x x^{\prime \prime} \cdot x^{\prime \prime} w^{\prime} \cdot \tilde{P}_{w^{\prime} w^{\prime \prime}} \cdot w^{\prime \prime} v \cdot P_{v x_{k}}$ is longer than $P$, a contradiction.

To finish the proof, suppose by contradiction that $\left|V(P) \cap \mathcal{C}_{X}\right|=1$ and $Y \in S_{x}$. Let $\left\{x^{\prime}\right\}=$ $V(P) \cap \mathcal{C}_{X}$. Since $|P| \geq 1$ and $V(P) \subseteq \mathcal{C}_{Y}^{X} \cup \mathcal{C}_{X}$, there exists an edge $x^{\prime} v$ in $P$ such that $v \in \mathcal{C}_{Y}^{X}$. This implies that $x$ is adjacent to both $x^{\prime}$ and $v$, a contradiction.

Lemma 35. Let $G$ be a connected full substar graph, $T$ be the host tree of an intersection model for $G$ and let $X$ be any vertex of $T$. If $\operatorname{lpt}(G)>1$, then there exists a longest path $P$ in $G$ and a node $Y \in N_{T}(X)$ such that $V(P) \subseteq \mathcal{C}_{Y}^{X}$.

Proof. We divide the proof in two cases, according to whether there exists a vertex in $G$ such that its corresponding substar is centered in $X$.

Case 1. $\mathcal{C}_{X} \neq \varnothing$

Let $x \in \mathcal{C}_{X}$. Moreover, suppose that $\left|S_{x}\right|$ is maximum over all such $x$. Since $\operatorname{lpt}(G)>1$, there exists a longest path $P$ in $G$ such that $x \notin V(P)$. By Lemma 34, there exists a node $Y$, adjacent to $X$ in $T$ such that $V(P) \in \mathcal{C}_{Y}^{X} \cup \mathcal{C}_{X}$. If $V(P) \cap \mathcal{C}_{X}=\varnothing$, the statement holds. Otherwise, $P$ has a vertex $x^{\prime}$ such that $x^{\prime} \in \mathcal{C}_{X}$. Also by Lemma 34 $V(P) \cap \mathcal{C}_{X}=\left\{x^{\prime}\right\}$. Note that $x^{\prime}$ is not an extreme of $P$, since $x \notin V(P)$. Moreover, if $N_{G}\left(x^{\prime}\right) \subseteq N_{G}(x)$, then $P$ would have to contain $x$. This implies that $d_{T}(X)-1 \leq\left|S_{x^{\prime}}\right| \leq\left|S_{x}\right|<d_{T}(X)$ and, as consequence, $\left|S_{x}\right|=\left|S_{x^{\prime}}\right|=d_{T}(X)-1$. That is, both $S_{x}$ and $S_{x^{\prime}}$ miss a node in the neighborhood of $X$. By Lemma 34, $Y \notin S_{x}$. Since $N_{G}\left(x^{\prime}\right) \nsubseteq N_{G}(x)$, we may assume that there exists $Z \in N_{T}(X)$ such that $Z \neq Y, Z \in S_{x}$ and $Z \notin S_{x^{\prime}}$.

Since $\operatorname{lpt}(G)>1$, there exists a longest path $Q$ in $G$ that does not contain $x^{\prime}$. By Lemma 34 , $V(Q) \subseteq \mathcal{C}_{Z}^{X} \cup\left\{x^{\prime \prime}\right\}$, for some $x^{\prime \prime} \in \mathcal{C}_{X}$. However, this implies that $P$ and $Q$ do not intersect each other, a contradiction with the fact that $G$ is connected. 
Case 2. $\mathcal{C}_{X}=\varnothing$

Let $K$ be the clique of $G$ formed by the vertices $x \in V(G)$ such that $X \in S_{x}$. We will show that if, for every longest path $P$, there is no $Y \in V(T)$ such that $V(P) \subseteq \mathcal{C}_{Y}^{X}$, then $\operatorname{lpt}(G)=1$. Suppose that every longest path $P$ of $G$ contains vertices whose substars are centered in two different branches of $T$ with respect to $X$. Since $\mathcal{C}_{X}=\varnothing, P$ must contain two consecutive vertices whose stars are centered in the neighborhood of $X$ in $T$. That is, $P$ has two consecutive vertices that belong to $K$ and therefore $P$ must contain all the vertices of $K$.

We are now ready to prove the main result of this section.

Theorem 36. If $G$ is a connected full substar graph, then $\operatorname{lpt}(G)=1$.

Proof. Suppose by a contradiction that $\operatorname{lpt}(G)>1$. Let $T$ be the host tree of an intersection model for $G$. We start by creating an auxiliary directed graph $D$ on the same vertex set as $T$ and arc set defined in the following way. For every $X \in V(D)$, we have that $X Y \in E(D)$ if $Y \in N_{T}(X)$ and there exists a longest path $P$ such that $V(P) \subseteq \mathcal{C}_{Y}^{X}$. By Lemma 35, every node in $T$ has outdegree at least one.

Let $X Y$ be the last arc in a maximal directed path in $D$. Since $T$ is a tree, $Y X$ is also an arc in $D$. Since $X Y \in E(G)$ and $Y X \in E(G)$, there exists two longest paths $P$ and $Q$ in $G$ such that $V(P) \in \mathcal{C}_{Y}^{X}$ and $V(Q) \in \mathcal{C}_{X}^{Y}$. However, since $\mathcal{C}_{X}^{Y} \cap \mathcal{C}_{Y}^{X}=\varnothing$, the paths $P$ and $Q$ do not have a vertex in common, a contradiction with the fact that $G$ is connected.

\section{CONClusion AND FUtURE WORK}

The problem of finding a minimum longest path transversal remains open for several well-studied graph classes. In this work, we proved that connected bipartite permutation graphs admit a transversal of size one. The problem remains open for connected biconvex graphs and connected permutation graphs, well-known superclasses of bipartite permutation graphs. Even though our upper bound for $\operatorname{lpt}(G)$, when $G$ is a connected chordal graph, depends on $\omega(G)$, so far there are no examples of connected chordal graphs that require a transversal of size greater than one. In this direction, one open problem is to look for such an example, if it exists, or to look for better bounds for $\operatorname{lpt}(G)$ when $G$ belongs to this graph class. Finally, it would be interesting to generalize Theorem 36 for the class of substar graphs, that is, intersection graphs of substars of a tree.

\section{REFERENCES}

[1] N. Alon, P. Seymour, and R. Thomas. A separator theorem for nonplanar graphs. J Am Math Soc, 3:801-808, 1990.

[2] P.N. Balister, E. Győri, J. Lehel, and R.H. Schelp. Longest paths in circular arc graphs. Combin. Probab. Comput., 13(3):311-317, 2004.

[3] H.L. Bodlaender. A partial $k$-arboretum of graphs with bounded treewidth. Theoretical Computer Science, 209(1):1-45, 1998.

[4] M.R. Cerioli and P. Lima. Intersection of longest paths in graph classes. Electronic Notes in Discrete Mathematics, 55:139-142, 2016. 
[5] F. Chen. Nonempty intersection of longest paths in a graph with a small matching number. Czechoslovak Mathematical Journal, 65(140):545-553, 2015.

[6] G. Chen, J. Ehrenmüller, C.G. Fernandes, C.G. Heise, S. Shan, P. Yang, and A.N. Yates. Nonempty intersection of longest paths in series-parallel graphs. Discrete Mathematics, 340(3):287-304, 2017.

[7] S.F. de Rezende, C.G. Fernandes, D.M. Martin, and Y. Wakabayashi. Intersecting longest paths. Discrete Mathematics, 313:1401-1408, 2013.

[8] R. Diestel. Graph Theory, 4th Edition, volume 173 of Graduate texts in mathematics. Springer, 2010.

[9] F.V. Fomin and D.M. Thilikos. New upper bounds on the decomposability of planar graphs. Journal of Graph Theory, 51(1):53-81, 2006.

[10] F. Gavril. The intersection graphs of subtrees in trees are exactly the chordal graphs. Journal of Combinatorial Theory, Series B, 16(1):47-56, 1974.

[11] G. Golan and S. Shan. Nonempty intersection of longest paths in $2 K_{2}$-free graphs. Available as $\operatorname{arXiv:1611.05967,2016.}$

[12] J.L. Gross. Embeddings of graphs of fixed treewidth and bounded degree. ARS Mathematica Contemporanea, 7:379-403, 2014.

[13] M. Heinz. Tree-decomposition: Graph minor theory and algorithmic implications. Master's thesis, Technischen Universität Wien, 2013.

[14] A.S. Jobson, A.E. Kézdy, J. Lehel, and S.C. White. Detour trees. Discrete Applied Mathematics, 206:73-80, 2016.

[15] F. Joos. A note on longest paths in circular arc graph. Discussiones Mathematicae Graph Theory, 35(3):419-426, 2015.

[16] S. Klavžar and M. Petkovšek. Graphs with nonempty intersection of longest paths. Ars Combinatoria, 29:43-52, 1990.

[17] P. T. Lima. Interseção de caminhos mais longos em grafos. Master's thesis, Rio de Janeiro: UFRJ/COPPE, 2016.

[18] D. Rautenbach and J.-S. Sereni. Transversals of longest paths and cycles. SIAM J. Discrete Math., 28(1):335-341, 2014.

[19] H. Walther and H.-J. Voss. Über Kreise in Graphen. VEB Deutscher Verlag der Wissenschaften, 1974.

[20] T. Zamfirescu. On longest paths and circuits in graphs. Math. Scand., 38(2):211-239, 1976. 